\title{
La politique de la santé cet été en Suisse
}

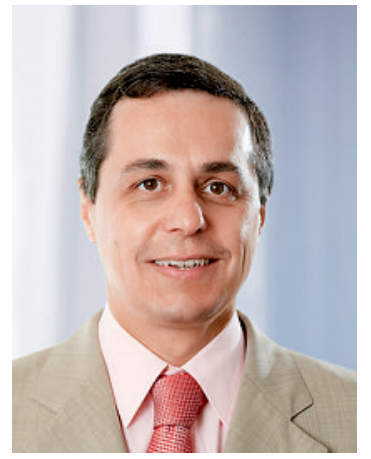

Ignazio Cassis
Un été chaud se prépare. Non, je ne parle ni de la météo, ni de la succession de Pascal Couchepin au Conseil fédéral. Je parle de la «politique de la santé», le mot que nous Suisses utilisons pour parler de la LAMal. La politique de la santé est en fait autre chose!

Nous sommes choqués encore une fois - par l'augmentation des primes de caisse maladie. Dans certains cantons, elles augmenteraient de 15 à $20 \%$, et l'on cherche un coupable! Oui, les coûts de la santé augmentent, surtout dans le secteur ambulatoire des hôpitaux et dans les EMS. L'augmentation a été globalement de $+4,9 \%$ en 2007 et de $+4,3 \%$ en 2008. Oui, les assureurs n'avaient pas prévu une telle augmentation, qui a plus que doublé par rapport aux années précédentes. Ils ont ainsi dû puiser dans les réserves, ce qui a entraîné une diminution d'environ $4 \%$ du taux de réserve en 2008. Même tendance pour 2009. Oui, la crise des marchés financiers a causé - comme pour d'autres assurances sociales ambulatoires hospitaliers ainsi qu'entre cantons, blocage du salaire du personnel médical, blocage de la rémunération pour les certificats d'incapacité au travail, participation aux coûts différenciés, etc.

Lors du débat urgent au Parlement le 10 juin dernier, les partis ont déploré à l'unisson le choc des primes et ont plus ou moins proposé tout ce qui était proposable, de l'abolition pure et simple de l'obligation de s'assurer à l'étatisation complète du système. Aucune décision n'a été prise. Lors de la séance de la Commission de la sécurité sociale et de la santé publique (CSSS-N) du 26 juin, les deux trains de mesures (celui du Conseil fédéral et celui du groupe de parlementaires) ont été discutés: j'ai été frappé par la légèreté avec laquelle la politique s'occupe de la matière. Des principes fondamentaux de la LAMal - tels que la différence structurelle entre tarifs d'office et tarifs négociés - pouvaient ainsi être hâtivement transformés en solutions mixtes qui ne sont ni chair ni poisson. Par exemple, le fait de donner au Conseil fédéral le droit d'intervenir d'autorité sur les tarifs négociés entre partenaires et approuvés par les gouvernements cantonaux équivaut - à mes yeux - à diluer la responsabilité de manière inacceptable. Finalement plus personne ne sera responsable et l'on finira par démotiver tout le monde.

\section{Le fait de donner au Conseil fédéral le droit d'intervenir d'autorité sur les tarifs né- gociés entre partenaires et approuvés par les gouvernements cantonaux équivaut à diluer la responsabilité de manière inacceptable}

(AVS, AI) - d'importantes pertes financières. Le besoin de rattrapage - face à une diminution du taux de réserve de 2,5\% en deçà du minimum légal de 11,5\% - occasionne à lui seul une augmentation des primes de $7 \%$ environ. Nous voici donc face à un défi, qui n'est ni nouveau ni différent de celui des autres pays de l'OCDE: freiner l'évolution des «coûts de la santé». Mais le choc populaire, amplifié par les médias, donne l'occasion aux politiciens de montrer qu'ils existent!

Le Conseil fédéral propose alors, le 29 mai, une modification de la LAMal intitulée «Mesures pour endiguer l'évolution des coûts». Je ne m'attarderai pas sur ces sept mesures, qui sont connues: conseil médical par téléphone, ticket modérateur, pilotage de l'offre dans le secteur ambulatoire hospitalier, élargissement des compétences du Conseil fédéral sur les tarifs, durée du rapport d'assurance, transmission des données des assureurs-maladie, augmentation extraordinaire des subsides de la Confédération. Quelque jours après, un groupe de parlementaires fédéraux avance d'autres propositions: le but déclaré est de ramener l'augmentation des primes pour 2009 à moins de $5 \%$. Dans ce train de mesures, on trouve un peu de tout: baisse des prix des médicaments et de leur coûts de distribution, utilisation «obligatoire» des génériques meilleur marché, réduction «manu militari» de la valeur du point TARMED pour réduire le clivage entre médecins praticiens et
L'été est encore long: la prochaine séance de la CSSS-N aura lieu le 27 août et le débat sera alors achevé. Toutes les propositions passeront à la CSSS du Conseil des Etats, qui en discutera le 2 septembre. Le Parlement décidera finalement en septembre, au cours de la session d'automne, et cette modification de la LAMal entrera en vigueur au $1^{\text {er }}$ janvier 2010, selon les règles du droit urgent.

Entre-temps la sous-commission LAMal de la CSSS-N continuera l'étude de la réforme de la médecine ambulatoire, qui se dirige vers une promotion du Managed Care. Nous y discutons actuellement des principes de base, pour passer ensuite à la modification de la loi.

Pour finir, je rappellerai quel lors de la votation finale de la session d'été, la prolongation du moratoire pour l'ouverture des cabinets médicaux a été approuvée par 107 contre 74

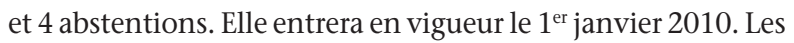
médecins de premiers recours sont exonérés du moratoire: tout médecin suisse ou provenant de l'Union européenne avec un titre de formation postgraduée en médecine générale, médecine interne ou pédiatrie, ou encore «médecin praticien», pourra s'installer et travailler à la charge des assurances sociales. L'été 2009 est décidément «hot»pour la santé!

Conseiller national et vice-président FMH 